Pacific Journal of Mathematics

ON BASES IN STRICT INDUCTIVE AND PROJECTIVE LIMITS 


\title{
ON BASES IN STRICT INDUCTIVE AND PROJECTIVE LIMITS OF LOCALLY CONVEX SPACES
}

\author{
Klaus Floret and Vincenzo Bruno Moscatelli
}

\begin{abstract}
This note investigates, for certain locally convex spaces which have bases and are strict inductive or projective limits, the structural property of being a direct sum or a product. Our approach is based on a suitably more general version of a decomposition lemma originally due to $S$. Dineen and gives a better understanding of the non-existence of bases in certain nuclear $(F)$ - and strict $(L F)$-spaces. Our method also allows us to investigate the structure of various other non-nuclear spaces with unconditional bases yielding, in particular, examples of spaces with no such bases. In part, this also motivated us to include some rather general remarks on the problem of when the strong dual of a homomorphism between locally convex spaces is a homomorphism as well.
\end{abstract}

There are nuclear spaces of type $(F)$ and proper strict $(L F)$ which do not admit bases. The first example of such a Fréchet space is due to Mitiagin and Zobin [18] in 1974. Later, in 1979, Dubinsky [6] constructed a Fréchet space (with a continuous norm) which does not even have the bounded approximation property; a very simple construction of spaces of this type was recently given by Vogt [23]. A totally different approach in constructing nuclear Fréchet spaces without bases was presented by the second author [19] in 1980. A nuclear, proper, strict ( $L F)$-space without a basis is exhibited in the paper [8] of the first author: this space has even the property that all stepspaces are nuclear Fréchet spaces with continuous norms and without the bounded approximation property. All the proofs have in common the fact that they show the non-existence of a basis by checking that the space under consideration does not have a property it should have if it had a basis, such as being countably normed [6], [23], being a product space [19], or having a continuous norm [8].

It is one of the purposes of this note ${ }^{1}$ to contribute to a better understanding of certain nuclear spaces of type $(F)$ and $(L F)$, with respect to admitting or not a basis, by using the structural property of being a product or a direct sum. At the same time, this approach will free the construction in [19] from a somehow not quite natural use of Dubinsky's classification of perfect Fréchet sequence spaces [5]. The key to our

\footnotetext{
${ }^{1}$ Part of the results were already presented at the $17^{\circ}$ Seminário de Análise [9].
} 
approach is a suitably more general version of a lemma due to Dineen ([4], 5.4.3).

Our method even allows us to investigate the structure of various other non-nuclear spaces with unconditional bases. In order to do this, we shall make, at the appropriate time ( $\$ 5)$, some general remarks on the problem of when the strong dual of a homomorphism is again a homomorphism.

\section{Preliminaries.}

1.1. The notation which we shall use is standard, e.g. as in [13]. In particular, an ( $L F)$-space (resp. an ( $L B)$-space) is the (locally convex) inductive limit of an increasing inductive sequence of Fréchet spaces (resp. of Banach spaces).

REMARK. Note that the inductive limit $E$ of a sequence of separated $(L F)$-spaces $E_{k}=\operatorname{ind}_{n \rightarrow} E_{k, n}$ is again an $(L F)$-space. Indeed, using Grothendieck's factorization theorem ([11], IV, §1.5) one can "thin out" the sequences to obtain the situation where $E_{k, n}$ embeds continuously into $E_{k+1, n}$ for all $k, n \in \mathbf{N}$. But then it is clear that $E=$ ind $_{k \rightarrow} E_{k, k}$.

An inductive sequence $\left(E_{k}\right)$ of locally convex spaces is called strict if $E_{k}$ is a topological subspace of $E_{k+1}$ for each $k \in \mathbf{N}$. For the properties of strict inductive sequences and their limits, see e.g. [10].

A projective sequence $\left(F_{k}\right)$ of locally convex spaces is called strict if all linking mappings $F_{k+1} \rightarrow F_{k}$ are open and onto. Of course, this is somehow the dual notion to that of a strict inductive sequence (see §5). Furthermore, a locally convex space is called twisted if it is not isomorphic to a product of locally convex spaces with continuous norms. Strict projective sequences of Banach and Fréchet spaces with twisted limit spaces were first constructed in [19] by the second author.

1.2. A basis $\left(e_{n}\right)$ of a locally convex space $E$ (always assumed to have continuous coefficient functionals $\varphi_{n}$, which is automatically true in $(F)$ and separated, locally complete ( $L F)$-spaces, [13], 14.3.4) is called unconditional if for every $x \in E$ the net

$$
\left(\sum_{n \in D}\left\langle\varphi_{n}, x\right\rangle e_{n}: D \subset \mathbf{N} \text { finite }\right)
$$

converges or, equivalently, if

$$
\sum_{n=1}^{\infty}\left\langle\varphi_{\pi(n)}, x\right\rangle e_{\pi(n)}=x
$$

for all permutations $\pi$ of $\mathbf{N}$. 
We shall use the following results:

(a) If $\left(e_{n}\right)$ is an unconditional basis of a complete space $E$, then all subseries of the series expansions of elements in $E$ converge.

(b) In barrelled spaces (such as $(F)$ - and separated ( $L F)$-spaces) which are nuclear, every basis is absolute, in particular, unconditional. This follows from the theorems of Banach-Steinhaus and Dynin-Mitiagin ([13], 21.10.1). Also, in nuclear $(F)$ - or complete $(D F)$-spaces the sequence of coefficient functionals is an absolute basis for the strong dual.

(c) A basis $\left(e_{n}\right)$ is unconditional if and only if $\left(e_{\pi(n)}\right)$ is a basis for all permutations $\pi$ of $\mathbf{N}$. (For a proof, use the biorthogonality of the coefficient functionals.)

1.3. For the purpose of this paper, a pair $(\mathfrak{D}, \mathfrak{R})$ of classes $\mathfrak{D}$ (for domain) and $\Re$ (for range) of separated locally convex spaces is called a closed-graph pair if it satisfies the following conditions:

(i) If $E \in \mathfrak{D}$ and $F \in \Re$, then every linear bijection $E \rightarrow F$ with closed graph is continuous.

(ii) $\Re$ is closed under the formation of closed subspaces and (locally convex) countable direct sums.

EXAMPLES.

(a) $\mathfrak{D}=$ ultrabornological spaces

$\Re=$ webbed spaces

(b) $\mathscr{D}=$ separated inductive limits of Baire spaces

$\Re=$ webbed spaces

(c) $\mathfrak{D}=$ ultrabornological spaces

$\Re=$ Souslin spaces,

(For a proof that the above pairs are closed-graph pairs, see e.g. De Wilde's monograph [3].)

\section{A general decomposition theorem.}

2.1. The following central result is Dineen's lemma ([4], 5.4.3) extended to its natural limits of validity.

TheOREM. Let $(\mathfrak{D}, \Re)$ be a closed-graph pair and let $\left(E_{k}\right)_{k \in \mathbf{N}}$ be a strict inductive sequence of complete spaces $E_{k} \in \Re$ such that ind in $_{k} E_{k}=$ : $E \in \mathfrak{D}$. If $E$ has an unconditional basis, then there are complemented subspaces $G_{k} \subset E_{k}$ with unconditional bases such that

$$
E=\bigoplus_{k=1}^{\infty} G_{k}
$$

algebraically and topologically. 
Proof. For $\left(e_{n}\right)$ an unconditional basis of $E$ and $\tau$ the topology of $E$ define, with $E_{0}:=\{0\}$,

$$
\begin{aligned}
& N_{k}:=\left\{n \in \mathbf{N}: e_{n} \in E_{k} \backslash E_{k-1}\right\}, \\
& G_{k}:=\overline{\operatorname{span}}\left\{e_{n}: n \in N_{k}\right\},
\end{aligned}
$$

where the closure is taken in $(E, \tau)$. Then $G_{k}$ is a closed subspace of $E_{k}$ and it is easy to check that:

(1) $\left(e_{n}: n \in N_{k}\right)$ is an unconditional basis of $G_{k}$,

(2) $G_{k} \cap G_{j}=\{0\}$ for $k \neq j$

and, since strict inductive sequences of complete spaces are sequentially retractive, i.e. every convergent sequence in $E$ converges in some $E_{k}$,

(3) $E=\oplus_{k=1}^{\infty} G_{k}$ algebraically.

Obviously, the identity map

$$
\bigoplus_{k=1}^{\infty}\left(G_{k},\left.\tau\right|_{G_{k}}\right) \rightarrow(E, \tau)
$$

is continuous and

$$
\left((E, \tau), \bigoplus_{k=1}^{\infty}\left(G_{k},\left.\tau\right|_{G_{k}}\right)\right) \in \mathfrak{D} \times \Re
$$

Therefore, (3) holds also topologically. Finally, since now each subspace $G_{k}$ is complemented in $E$, it is complemented in $E_{k}$ as well.

2.2. Each one of the three examples of closed-graph pairs in $\S 1$ implies the following

Corollary. Let $\left(E_{k}\right)_{k \in \mathrm{N}}$ be a strict inductive sequence of separated, complete ( $L F$ )-spaces. Then $E=$ ind $_{k \rightarrow} E_{k}$ has an unconditional basis (if and) only if there are complemented subspaces $G_{k} \subset E_{k}$ with unconditional bases such that

$$
E=\bigoplus_{k=1}^{\infty} G_{k}
$$

as locally convex spaces.

In general, subspaces of ( $L F)$-spaces need not be $(L F)$-spaces, but here the $G_{k}$ 's are complemented and, therefore, $(L F)$-subspaces. Applying the corollary to the case when the spaces $E_{k}$ are nuclear Fréchet, we recover Dineen's original result.

\section{Bases in strict inductive limits.}

3.1. As a first application to the existence problem for bases we look at the strict $(L B)$-spaces constructed in [19], $\$ 1$ : by Lemma 3 of that paper they are not isomorphic to a direct sum of Banach spaces, whence 
(by Corollary 2.2) cannot have an unconditional basis and we obtain

Proposition. Strict (LB)-spaces that are not isomorphic to a direct sum of Banach spaces (in particular, the spaces of [19], §1) do not have an unconditional basis.

3.2. There are reflexive Banach spaces $X$ with uncomplemented subspaces $Y$, both having unconditional bases. For an example, take $X=$ $L^{p}(0,1)$ and (according to [14]) a subspace $Y \subset X$ isomorphic to $l^{q}$, with $1<p \neq q<2$. If $Y$ were complemented in $X$, then it would contain a subspace isomorphic to $l^{p}$ (by [15], Corollary 3, p. 168) and this is not possible by a classical result of Banach ([1], Ch. XII, Théorème 7), saying that $l^{p}$ is not a subspace of $l^{q}$. For examples of uncomplemented subspaces of $l^{p}$ with unconditional bases, see [17], p. 91. Now, using a pair $(X, Y)$ of this kind in the construction of [19], $\S 1$, it is straightforward to check that each space $(X \oplus \cdots \oplus X \oplus Y \oplus Y \oplus \cdots)_{l^{2}}$ has an unconditional basis and hence we have the

COROLlary. There is a strict inductive sequence of reflexive Banach spaces with unconditional bases the limit of which has no unconditional basis.

3.3. In the setting of $(L F)$-spaces, part of the following was already used in [8]:

Proposition. If $\left(E_{k}\right)$ is a strict inductive sequence of separated and complete (LF)-spaces with continuous norms and $E:=\operatorname{ind}_{k \rightarrow} E_{k}$ has no continuous norm (a sequence of nuclear Fréchet spaces $E_{k}$ of this kind was first constructed in [8], §5), then $E$ has no unconditional basis and hence no basis at all if $E$ is nuclear.

Proof. If $E$ had an unconditional basis then, by Corollary 2.2, it would be the direct sum of subspaces $G_{k}$ of $E_{k}$, each having a continuous norm $p_{k}$ : but then $\sum_{k=1}^{\infty} p_{k}$ would be a continuous norm on $\bigoplus_{k=1}^{\infty} G_{k}=$ E.

\section{Bases in nuclear Fréchet spaces.}

4.1. Concerning the structure of certain nuclear Fréchet spaces, the following result holds:

Proposition. Let $F$ be a Fréchet space which is the limit of a strict projective sequence $\left(F_{k}\right)$ of nuclear Fréchet spaces. Then $F$ has a basis (if and) only if it is isomorphic to the product of a sequence of complemented subspaces $H_{k}$ of $F_{k}$ with a basis. 
Proof. Since $F$ is a reflexive Fréchet space, its strong dual $F_{\beta}^{\prime}$ is bornological, hence $F_{\beta}^{\prime}=\operatorname{ind}_{k \rightarrow}\left(F_{k}\right)_{\beta}^{\prime}$ and this inductive limit is strict, as well known (see also the next section). If $F$ has a basis, then also $F_{\beta}^{\prime}$ has a basis and Corollary 2.2 gives that $F_{\beta}^{\prime}$ is a direct sum of complemented subspaces of $\left(F_{k}\right)_{\beta}^{\prime}$ with basis. Dualizing this, we obtain the conclusion.

\subsection{The above immediately implies the}

COROLlaRY. The twisted, nuclear Fréchet spaces of [19], §2 do not admit a basis.

Originally this result was proved in [19] by using Dubinsky's classification of perfect Fréchet sequence spaces [5].

4.3. In the light of Proposition 4.1 and Corollary 2.2, it is very natural to construct bases in nuclear spaces that are strict projective or inductive limits (such as $\mathbb{E}(\Omega)$ and $\mathfrak{D}(\Omega)$ ) via representations as products or direct sums (see Vogt's paper [22]). Note that, by results of Valdivia [21], all the spaces $\&(\Omega)$ are isomorphic and the same is true for all the spaces $\mathscr{D}(\Omega)$. Moreover, once bases are known, the present results yield representations in terms of explicit subspaces.

\section{Strong duals of homomorphisms.}

5.1. In order to extend Corollary 4.2 to a more general setting, we need to investigate the question of when the strong dual $T_{\beta}^{\prime}: F_{\beta}^{\prime} \rightarrow E_{\beta}^{\prime}$ of a homomorphism $T: E \rightarrow F$ between locally convex spaces is again a homomorphism (a homomorphism is a continuous map which is open onto its image). On this question there are many results around, some of them folklore, but it still seems worthy to make some other systematic statements than those made in [16], $\$ 32$ and 33. Indeed, despite their extreme simplicity, our statements yield results which are surprisingly much more general than many published so far.

Since every homomorphism $T$ of $E$ into $F$ factors in a obvious way:

$$
E \rightarrow E / \operatorname{ker} T \stackrel{\cong}{\rightarrow} T(E) \rightarrow F,
$$

we shall treat the cases of $T$ being 1-1 and onto separately, since they are totally different in character.

5.2. The case of $T$ being 1-1 amounts, of course, to checking when the strong dual of a subspace of a locally convex space carries the quotient topology of the strong dual of the space. This question was investigated in 
[7]: if $\mathfrak{H}_{E}(0)$ is a basis of neighbourhoods of zero in $E$, then the inductive topology $\iota$ of $E^{\prime}$ is defined by

$$
E_{\iota}^{\prime}=\operatorname{ind}_{U \in \mathfrak{u}_{E}(0)} \llbracket U^{0} \rrbracket,
$$

where $\llbracket U^{0} \rrbracket$ is the Banach space spanned by $U^{0}$ and normed by the Minkowski functional of $U^{0}$. Then the results and proofs of [7] yield the

Proposition. (1) For every locally convex space $E$ the following assertions are equivalent:

(a) $E_{\iota}^{\prime}=E_{\beta}^{\prime}$.

(b) For every isomorphism $T$ of $E$ into any locally convex space $G$, the dual $T_{\beta}^{\prime}$ is a homomorphism.

(c) The strong dual of the embedding map of $E$ into any canonical product of Banach spaces (relative to a neighbourhood basis of zero) is a homomorphism.

(2) If in $E^{\prime}$ every strong null-sequence is equicontinuous, then (a)-(c) are equivalent to:

(d) $E_{\beta}^{\prime}$ is bornological.

(3) If $E$ is a metrizable space, then (a)-(d) are equivalent to

(e) $E$ is distinguished.

(f) The same as (b) with $G$ a Fréchet space.

In particular, if $E$ is a ( $g D F$ )-space, then (a)-(c) hold by (2). Of course, the assumption of (2) is satisfied if $E$ is quasi-barrelled. But note that if $E$ is a Montel space (hence barrelled and distinguished) which is not complete, then $E_{\beta}^{\prime}$ is not bornological and, therefore, (1c) is violated, which, by the way, contradicts a statement at the end of [11], Ch. IV, §2.4. (engl. edition p. 164).

5.3. Since the Mackey topology respects quotients, the following is obvious.

Proposition. The strong dual of any isomorphism of a locally convex space into a semi-reflexive locally convex space with a closed range is a homomorphism.

5.4. For the onto case, the general situation is much simpler; indeed, a straightforward use of polarity arguments yields the well-known (see e.g. [16] p. 11):

Proposition. Let $T: E \rightarrow F$ be continuous. Then $T_{\beta}{ }^{\prime}$ is an isomorphism (into) if and only if every bounded subset $B \subset F$ can be T-lifted to a bounded set $C \subset E$, i.e. $\overline{T(C)} \supset B$. 
Note that $T$ has dense range, necessarily.

The lifting property implies that $T$ is nearly open if $F$ is quasi-barelled. Pták's open mapping theorem has, therefore, the following consequence.

Corollary. Let $E$ be B-complete, let $F$ be quasi-barrelled and let $T$ : $E \rightarrow F$ be continuous. If $T_{\beta}^{\prime}$ is an isomorphism (into), then $T$ is open, in particular, onto.

This generalizes a classical result of Dieudonné and Schwartz for Fréchet spaces $E$ and $F$ (see e.g. [12], p. 310).

\subsection{The results in $\$ \S 5.2-5.4$ imply the}

THEOREM. If T: $E \rightarrow F$ is a homomorphism such that:

(a) every bounded set in $E / \operatorname{ker} T$ can be lifted (with respect to the quotient map).

(b) $(E / \operatorname{ker} T)_{\iota}^{\prime}=(E / \operatorname{ker} T)_{\beta}^{\prime}$ or: $F$ is semi-reflexive and has closed range, then $T_{\beta}^{\prime}$ is a homomorphism.

Some special cases when this theorem applies are collected in the following

COROLlaRY. The strong dual of a homomorphism $T: E \rightarrow F$ is a homomorphism in each of the following cases:

(1) E Fréchet and every bounded set in $F$ is precompact;

(2) E Fréchet-Schwartz, $F$ an arbitrary locally convex space;

(3) E a ( $g D F)$-space, $F$ an arbitrary locally convex space;

(4) $E$ a separated ( $L F)$-space, $F$ the inductive limit of a regular sequence of Fréchet-Montel spaces and $T$ with closed range;

(5) $E=\mathfrak{D}\left(\Omega_{1}\right), F=\mathfrak{D}\left(\Omega_{2}\right)$ and $T$ with closed range.

Proof. (1) and (2) follow from the facts that compact sets in quotients of Fréchet spaces can be compactly lifted and that reflexive Fréchet spaces are distinguished. Also, since quotients of ( $g D F)$-spaces are $(g D F)$ and bounded sets can be lifted ([13], p. 261), case (3) follows from Proposition 5.2.(2). To see (4), note first that $F$ is reflexive. So it remains to show that every bounded set in $E / \operatorname{ker} T$ can be lifted. Take inductive sequences $\left(E_{n}\right)$ of Fréchet spaces and $\left(F_{n}\right)$ of Fréchet-Montel spaces defining $E$ and $F$ respectively. By the closed graph theorem for $(L F)$ spaces, it is easily seen that

$$
E / \operatorname{ker} T=\operatorname{ind}_{n \rightarrow} E_{n} /\left[E_{n} \cap \operatorname{ker} T\right] \simeq \operatorname{ind}_{n \rightarrow}\left[F_{n} \cap T(E)\right]
$$


holds topologically. If $B \subset E / \operatorname{ker} T$ is bounded, then $T(B)$ is compact in some $F_{m} \cap T(E)$. It follows from Grothendieck's factorization theorem ([11], IV, §1.5) that

$$
F_{m} \cap T(E) \rightarrow E_{n} / E_{n} \cap \operatorname{ker} T
$$

continuously for some $n$, so that $B$ is relatively compact in $E_{n} / E_{n} \cap \operatorname{ker} T$ and can be lifted to $E_{n}$ and hence to $E$. Finally (5) is a special case of (4).

This generalizes various known results. Note that if $E$ is a FréchetMontel space with a quotient isomorphic to $l^{p}$ (see [16], pp. 22-23), then, obviously, the strong dual of the quotient map is not a homomorphism.

5.6. Clearly, these results provide many situations in which the strong dual of a strict projective (resp. inductive) sequence is a strict inductive (resp. projective) sequence.

\section{Unconditional bases in certain Fréchet spaces.}

6.1. The dual-homomorphism Theorem 5.5. enables us to extend the results of $\S 4$ on bases in nuclear Fréchet spaces to the following

Proposition. Let the locally convex space $F$ be the limit of a strict projective sequence $\left(F_{k}\right)$. If

(a) each $F_{k}$ is a Fréchet-Montel space, or

(b) each $F_{k}$ is a reflexive ( $D F$ )-space,

then $F$ has an unconditional basis (if and) only if $F$ is isomorphic to the product of a sequence of complemented subspaces $H_{k}$ of $F_{k}$ with an unconditional basis.

Proof. 1. Let us assume for the moment that $F$ is reflexive and

$$
F_{\beta}^{\prime}=\operatorname{ind}_{k \rightarrow}\left(F_{k}\right)_{\beta}^{\prime} \text {. }
$$

The inductive sequence of the strong duals is strict in both cases (a) and (b) by 5.5 . By the remark in 1.1 , the space $F_{\beta}^{\prime}$ is, therefore, a reflexive $(L F)$-space. Now take an unconditional basis in $F$; since the coefficient functionals form a weak ${ }^{*}=$ weak basis in $F^{\prime}$ and in separated $(L F)$-spaces the weak-basis theorem holds $([13], 14.3 .5)$, the strong dual $F_{\beta}^{\prime}$ has an unconditional basis by 1.2.(c). Then Corollary 2.2 implies that there are complemented subspaces $G_{k}$ of $\left(F_{k}\right)_{\beta}^{\prime}$ with an unconditional basis such that

$$
F_{\beta}^{\prime}=\bigoplus_{k=1}^{\infty} G_{k}
$$


It follows that

$$
F=F_{\beta}^{\prime \prime}=\prod_{k=1}^{\infty}\left(G_{k}\right)_{\beta}^{\prime}
$$

and, since the $G_{k}$ 's are complemented, each $\left(G_{k}\right)_{\beta}^{\prime}$ can be identified with a complemented subspace $H_{k}$ of $F_{k}$. By the same reasoning as before, each $H_{k}$ has an unconditional basis.

2. It remains to prove the relation $(*)$.

(a) $F$ is clearly reflexive and a Fréchet-space, so its strong dual is bornological and, therefore, $(*)$ holds.

(b) Denoting the mapping $F_{k} \rightarrow F_{k-1}$ by $T_{k}$, it follows from the reflexitivity that for every bounded and closed $B_{k-1} \subset F_{k-1}$, there is a bounded and closed $B_{k} \subset F_{k}$ such that $T_{k}\left(B_{k}\right)=B_{k-1}$. This implies, by the very definition of a projective limit, that the canonical map $F \rightarrow F_{k}$ lifts all bounded sets (in the sense of 5.4), whence $\left(F_{k}\right)_{\beta}^{\prime}$ is a subspace of $F_{\beta}^{\prime}$. Since $F$ is semiflexive, $F_{\beta}^{\prime}$ is barrelled and, therefore, a result of Valdivia ([20], Cor. 1.5.) yields (*). To see that $F$ is even reflexive take a weak* ${ }^{*}$ weak bounded subset $B \subset F^{\prime}$ : it is bounded in $F_{\beta}^{\prime}$ and so bounded in some $\left(F_{k}\right)_{\beta}^{\prime}$ since the inductive limit is strict; therefore $B$ is equicontinuous in $F_{k}^{\prime}$ and hence in $F^{\prime}$.

The last part of the proof showed that the limit of a strict projective sequence of reflexive $(D F)$-spaces is reflexive.

COROllary. The reflexive, twisted Fréchet spaces which were constructed in [19], §1, Theorem 1, do not have unconditional bases. In particular (dualizing Corollary 3.2), there is a Fréchet space without an unconditional basis which is the strict projective limit of reflexive Banach space with an unconditional basis.

The first part of this result can also be obtained by using Dubinsky's classification of perfect Fréchet sequence spaces, since every reflexive Fréchet space with an unconditional basis is a perfect sequence space, because the basis is then boundedly complete (see also [2], §5).

\section{REFERENCES}

[1] S. Banach, Théorie des opérations linéaires, Warsaw 1932.

[2] S. F. Bellenot and E. Dubinsky, Fréchet spaces with nuclear Köthe quotients, Trans. Amer. Math. Soc., 273 (1982), 579-594.

[3] M. De Wilde, Closed Graph Theorems and Webbed Spaces, Pitman Research Notes Math., 19 (1978). 
[4] S. Dineen, Complex Analysis in Locally Convex Spaces, North-Holland Math. Studies, 57 (1981).

[5] E. Dubinsky, Perfect Fréchet spaces, Math. Ann., 174 (1967), 186-194.

[6] The Structure of Nuclear Fréchet Spaces; Springer-Lect. Notes Math. 720 (1979).

[7] K. Floret, Über den Dualraum eines lokalkonvxen Unterraums, Arch. Math., 25 (1974), 646-648.

[8] Continuous norms on locally convex strict inductive limit spaces, (to appear in Math. Z.).

[9] K. Floret and V. B. Moscatelli, On bases and decompositions in (LF)-spaces, $17^{\circ}$ Sem. Bras. Análise, (1983), 169-175.

[10] K. Floret and J. Wloka, Einführung in die Theorie der lokalkonvexen Räume, Springer Lect. Notes Math., 56 (1968).

[11] A. Grothendieck, Espaces vectoriels topologiques, São Paulo, 1964.

[12] J. Horvath, Topological Vector Spaces and Distributions I; Addison Wesley, 1966.

[13] H. Jarchow, Locally convex spaces, Teubner, 1981.

[14] M. I. Kadec, On the linear dimension of the spaces $L_{p}$ and $l_{q}$, Usp. Math. Nauk, 13 (1958), 95-98 (Russian).

[15] M. I. Kadec and A. Pelczyński, Bases, lacunary sequences and complemented subspaces in the spaces $L_{p}$, Studia Math., 21 (1961/62), 161-176.

[16] G. Köthe, Topological Vector Spaces II, Springer, 1979.

[17] J. Lindenstrauss and L. Tzafriri: Classical Banach Spaces I, Springer, 1977.

[18] B. S. Mitiagin and N. M. Zobin, Contre-exemple à l'existence d'une base dans un espace de Fréchet nucléaire; C. R. A. S. Paris, 279 A (1974), 255-256 and 325-327.

[19] V. B. Moscatelli, Fréchet spaces without continuous norms and without bases, Bull. London Math. Soc., 12 (1980), 63-66.

[20] M. Valdivia, Absolutely convex sets in barrelled spaces, Ann. Inst. Fourier, 21 (1971), 3-13.

[21] , Representaciones de los espacios $\mathfrak{D}(\Omega)$ y $\mathfrak{D}^{\prime}(\Omega)$, Rev. Real Acad. Cienc. Exact. Fís. Natur. Madrid, 72 (1978), 385-414.

[22] D. Vogt, Sequences space representations of spaces of test functions and distributions, Funct. Anal., Holomorphy and Approx. Theory (ed. G. Zapata), Marcel Dekker Lect. Notes Pure Appl. Math., 83 (1983), 405-443.

[23] An example of a nuclear Fréchet space without the bounded approximation property, Math. Z., 182 (1983), 265-267.

Received November 23, 1983. This work was done while the authors were visiting, respectively, the Universidade Federal do Rio de Janeiro and the Universidade Federal Fluminense, during 1983.

FACHBEREICH MATHEMATIK / INF. DER UNIVERSITÄT

D-2900 OLDENBURG

WEST GERMANY

AND

UNIVERSITÀ - C. P. 193

73100 LECCE

ITALY 



\section{PACIFIC JOURNAL OF MATHEMATICS EDITORS}

\author{
V. S. VARADARAJAN (Managing Editor) \\ University of California \\ Los Angeles, CA 90024 \\ Charles R. DePrima \\ California Institute of Technology \\ Pasadena, CA 91125 \\ R. FINN \\ Stanford University \\ Stanford, CA 94305
}

\author{
HeRmanN FlaschKa \\ University of Arizona \\ Tucson, AZ 85721 \\ Ramesh A. Gangolli \\ University of Washington \\ Seattle, WA 98195 \\ ROBION KIRBY \\ University of California \\ Berkeley, CA 94720
}

C. C. MOORE

University of California

Berkeley, CA 94720

H. SAMELSON

Stanford University

Stanford, CA 94305

HAROLD STARK

University of California, San Diego

La Jolla, CA 92093

ASSOCIATE EDITORS
R. ARENS
E. F. BECKENBACH
B. H. NeUmanN
F. WOLF
K. YosHIDA (1906-1982)

\section{SUPPORTING INSTITUTIONS}

UNIVERSITY OF ARIZONA

UNIVERSITY OF BRITISH COLUMBIA

CALIFORNIA INSTITUTE OF TECHNOLOGY

UNIVERSITY OF CALIFORNIA

MONTANA STATE UNIVERSITY

UNIVERSITY OF NEVADA, RENO

NEW MEXICO STATE UNIVERSITY

OREGON STATE UNIVERSITY
UNIVERSITY OF OREGON

UNIVERSITY OF SOUTHERN CALIFORNIA

STANFORD UNIVERSITY

UNIVERSITY OF HAWAII

UNIVERSITY OF TOKYO

UNIVERSITY OF UTAH

WASHINGTON STATE UNIVERSITY

UNIVERSITY OF WASHINGTON 


\section{Pacific Journal of Mathematics}

Vol. 119, No. $1 \quad$ May, 1985

Maurice Chacron, Nonisotropic unitary spaces and modules with

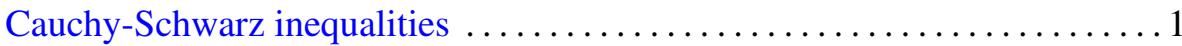

Myriam Dechamps-Gondim, Françoise Piquard and H. Queffélec, On

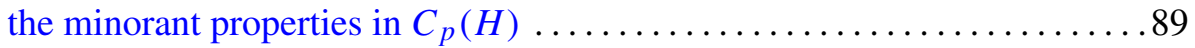

Klaus Floret and V. B. Moscatelli, On bases in strict inductive and projective limits of locally convex spaces .................... 103

Norman Joseph Goldstein, Degenerate secant varieties and a problem on

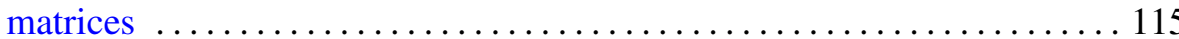

Harold Morris Hastings and Stefan Waner, $G$-bordism with singularities

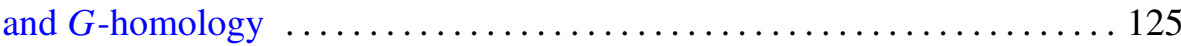

Takesi Isiwata, Clopen realcompactification of a mapping ........... 153

Hisao Kato, Concerning hyperspaces of certain Peano continua and strong

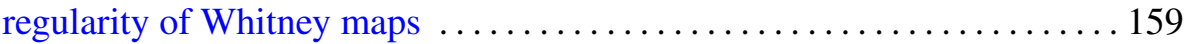

Elyahu Katz and Sidney Allen Morris, Free products of topological groups

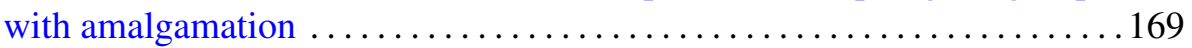

Kyewon Koh Park, Nice dense subsets for ergodic flows and Bernoulli

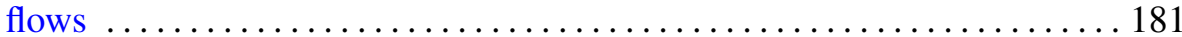

Francis Pastijn and Peter George Trotter, Lattices of completely regular

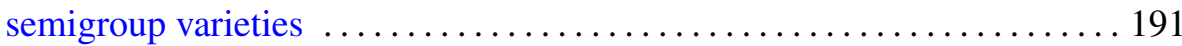

Rae Michael Andrew Shortt, Reticulated sets and the isomorphism of analytic powers

David A. Stegenga and Kenneth R. Stephenson, Generic covering properties for spaces of analytic functions

M. V. Subba Rao and R. Sitaramachandra Rao, On some infinite series of

L. J. Mordell and their analogues 